à la constitution du corpus d'étude pouvait sans doute permettre d'intégrer des passages bien délimités de ces deux œuvres évangéliques majeures. Au total, cet échantillon est représentatif et suffisant pour mener des analyses stylistiques précises. Il est sans doute un peu frêle pour généraliser les conclusions qu'on peut en tirer à l'ensemble du mouvement évangélique. De plus, ce corpus n'est pas systématiquement comparé à d'autres ensembles de référence : il serait intéressant de généraliser les rapprochements ponctuels qui sont consentis avec les textes de Luther, et de comparer plus longuement les textes évangéliques avec les textes catholiques de la même période : quel emploi font alors les théologiens conservateurs des adjectifs en question ou de leurs correspondants latins ? Un détour plus systématique par le corpus adverse pourrait permettre de valider un certain nombre d'hypothèses qui sont faites dans cette étude sur la spécificité des tours de langages affectionnés par les Évangéliques.

Il faut sans doute placer l'intérêt de ce travail non pas dans la confirmation ou non de l'existence d'un « réseau » évangélique et dans sa délimitation (ce qui reste du ressort de l'histoire et de ses méthodes d'investigation), mais dans la mise au point très appréciable d'outils à la fois théoriques et contextuels de pragmatique historique, qui permettent de mieux comprendre comment quelques personnes partageant une croyance peuvent créer une langue apte à les réunir, à fédérer leur combat et à diffuser leur pensée. Au total, cette belle enquête apporte un éclairage nouveau et précis sur la stylistique de l'adjectif dans les textes de la Renaissance ; elle tisse aussi des liens inédits entre théologie et littérature. On ne lira plus les épithètes de Marguerite ou de Marot de la même manière.

ALEXANDRe TARRÊTE, Université de Paris IV - Sorbonne

\title{
Mireille Huchon.
}

Le français au temps de Jacques Cartier.

Rimouski : Tangeance, 2006, $93 \mathrm{p}$.

Dans Le français au temps de Jacques Cartier, M. Huchon présente une étude linguistique et stylistique du Brief recit, un ouvrage qui relate le second voyage de Jacques Cartier en Nouvelle-France - un ouvrage riche d'enseignements sur le plurilinguisme et la variation linguistique au $\mathrm{XVI}^{\mathrm{e}}$ siècle que l'érudite française nous décortique avec un rare bonheur. Publié en français à Paris en 1545, ce récit n’a pas joui d'un grand rayonnement, si bien qu'en 1609, Marc Lescarbot soulignait déjà l'oubli dans lequel le récit était tombé. Il aurait probablement eu plus de succès s'il 
avait été écrit en latin, car si le $\mathrm{XvI}^{\mathrm{e}}$ siècle est une période où l'emploi du vernaculaire commence à jouir d'une certaine légitimité dans de nombreuses sphères en France, telles que la justice et la religion, le latin conservait son statut de langue d'érudition et son caractère universel permettait mieux la diffusion d'une œuvre littéraire dans de nombreux pays d'Europe.

L'ouvrage se divise en neuf sections, qu'on peut regrouper en deux parties : la première présente l'état de la langue française dans lequel s'inscrit le Brief recit, avec exemples à l'appui. La seconde partie aborde l'aspect littéraire du récit dans son contexte culturel et accorde une attention particulière au premier lexique (langue amérindienne/français publié) qui accompagne ce texte du corpus précolonial.

L'auteure commence par mettre en évidence, dans la première partie, la présence constante d'autres langues, savantes et vernaculaires, dans les ouvrages de réflexion et de codification sur la langue française à la Renaissance (Calepinus 1502 ; Palsgrave 1530 ; Sylvius 1531 ; Estienne 1539 ; Calepinus 1585 ; Duret 1613), en soulignant l'importance des variations interlinguistiques (rapports entre langues officielle, véhiculaire, maternelle, seconde et de culture) et intralinguistique (utilisation de variantes d'un même système linguistique).

La concurrence entre latin et français, pour le statut de langue officielle et de langue des échanges, et entre français et dialectes, pour la langue maternelle, est ensuite présentée. La diversité des formes et des prononciations découlant de l'universalité du latin, universalité appuyée par une citation du Brief recit, a conféré au latin un statut ambigu qui a eu pour rôle, indique l'auteure, d'enrichir le vocabulaire français, alors que les humanistes tentaient de redonner au latin sa prononciation originelle et prenaient position dans la polémique autour de la publication d'œuvres religieuses en langue française.

Le texte de Jacques Cartier permet à l'auteure d'illustrer l'importance des emprunts à l'italien dans la langue française à la Renaissance, comme cela est bien connu et attesté. Après un aperçu du débat sur la «prééminence du français ou du toscan $\gg(38)$ au XVI ${ }^{\mathrm{e}}$ siècle, $\mathrm{M}$. Huchon rappelle les principaux champs d'activités et domaines touchés par l'italianisme, et l'existence de deux langues de la marine en France : celle du Levant, en Méditerranée, italianisée, et celle du Ponant, en Atlantique, ce qui explique la faible fréquence de termes de la navigation empruntés à l'italien dans l'ouvrage de l'explorateur malouin.

L’hétérogénéité de la langue d'oïl, malgré un « fonds important de mots communs » (40), amène l'auteure à aborder l'importance et la valorisation des dialectes dans l'enrichissement de la langue française. Les opinions divergentes sur ce rapport 
entre français et dialectes sont présentées dans le cadre du débat sur la langue parlée par les colons en Nouvelle-France et sur la constitution du français canadien.

La première partie de ce livre se termine par une description des variations graphiques courantes $\mathrm{au} \mathrm{XvI}^{\mathrm{e}}$ siècle et celles qui sont dues au caractère arbitraire des systèmes orthographiques des auteurs et des imprimeurs. Les principales correspondances entre graphie et prononciation sont bien exemplifiées et détaillées. Bien que brèves, les remarques d'ordre lexical soulignent l'un des traits les plus importants de la langue du $\mathrm{XvI}^{\mathrm{e}}$ siècle, l'usage abondant des synonymes. Quant aux remarques sur la syntaxe, elles se limitent à la concurrence entre les démonstratifs celui et icelui dans la langue et à l'emploi des formes non et nenny dans le Brief recit pour illustrer la « concurrence des systèmes et des interférences » (49).

La seconde partie du livre examine d'abord le Brief recit dans le contexte des débats français et italien sur le développement de la langue littéraire. M. Huchon souligne le petit nombre de procédés stylistiques du récit (comparaison et énumération) et la répétitivité des structures, indiquant ainsi la primauté de la fonction informative. La « conception utilitaire de la langue » du Brief recit (54) se traduit par l'absence de réflexion sur l'origine, la nature et la diversité des langues, thèmes souvent abordés au cours de ce siècle par les humanistes, et se manifeste par les pratiques d'attribution des noms propres aux lieux visités par les Français. Son analyse des aspects de la communication entre les Français et les « Sauvages » relève la qualité de la narration des scènes impliquant les interprètes de Cartier et révèle le doute qui s'y exprime quant à la fidélité des traductions.

La relation du second voyage de Cartier comprend un lexique français/amérindien, dont le fac-similé est présenté en annexe (79-83). Après une introduction à la nomenclature de ce lexique, $M$. Huchon souligne qu'on ne retrouve pas, dans cette liste de mots, tous les termes en langue amérindienne utilisés par Cartier dans son Brief recit. À ce caractère incomplet s'ajoutent des équivalences qualifiées d'insolites par l'auteure. De plus, en 1556, dans sa traduction en italien du récit du premier voyage de Cartier, Ramusio a donné un lexique italien/amérindien dont les formes sont complètement différentes de celles présentées dans le Brief recit. Enfin, Marc Lescarbot, qui a passé un an en Acadie en 1603, a relevé l'absence sur le terrain des mots listés dans le lexique du Brief recit; son lexique, qui reprend les mêmes concepts que celui de Cartier, présente lui aussi des formes différentes en langue amérindienne. Ces faits amènent l'auteure à examiner les problèmes posés par le lexique inclus dans le Brief recit en 1545 : à quel moment a-t-il été écrit, quelle est la langue décrite, qui furent les informateurs et le(s) véritable(s) rédacteur(s). 
Elle conclut l'ouvrage en s'interrogeant sur la fiabilité et les enjeux de ce récit du second voyage de Cartier.

Ce petit livre situe bien le récit de Jacques Cartier dans la perspective linguistique/littéraire du $\mathrm{XvI}^{\mathrm{e}}$ siècle. La présentation aurait certainement gagné en clarté si l'auteure avait précisé, dès le début du livre, sa définition du terme « français » : un passage sur la langue française au Canada suppose que le français commun de cette époque est le « français courant alors en usage en Île-de-France » (44). L'ouvrage aborde en effet la concurrence entre français et dialectes dans une langue d'oïl hétérogène, que soulignent les grammairiens, au $\mathrm{XVI}^{\mathrm{e}}$ siècle, en multipliant « les références aux parlers régionaux, Sylvius à celui de Picardie, Meigret à celui du Lyonnais » (Mireille Huchon, Le français de la Renaissance [Paris : Presses Universitaires de France, 1998, coll. Que sais-je?], p. 18). La précision du sens donné à « français » aurait ainsi contribué à rendre plus nette l'opposition avec « dialecte $\gg$. La brièveté de ce livre (93 pages) suggère de prime abord qu' il est destiné à un public non initié, auquel l'auteure réussit merveilleusement bien à expliquer, à l'aide d'exemples modernes, des concepts aussi complexes que la variation inter- et intralinguistique ou que le rapport entre dialectes et français. La pertinence des résumés et leur documentation seront aussi très appréciées par les linguistes, littéraires et historiens qui souhaitent se préparer à la lecture d'œuvres du XVI ${ }^{\mathrm{e}}$ siècle et d'ouvrages linguistiques plus approfondis sur cet état de la langue française. Encore une fois, Mireille Huchon lie admirablement vulgarisation et synthèse scientifique dans un ouvrage accessible aux néophytes comme aux spécialistes. 